Supporting Information for

\title{
Engineered Biosynthesis of Plant Polyketides: Manipulation of Chalcone Synthase
}

\author{
Ikuro Abe, $*, \dagger, \Phi$ Tatsuya Watanabe ${ }^{\dagger}$ Hiroyuki Morita ${ }^{\ddagger}$ Toshiyuki Kohno, ${ }^{\ddagger}$ Hiroshi Noguchi, $\dagger$ \\ $\dagger$ School of Pharmaceutical Sciences and the COE 21 Program, \\ University of Shizuoka, Shizuoka 422-8526, Japan \\ I PRESTO, Japan Science and Technology Agency, Kawaguchi, \\ Saitama 332-0012, Japan \\ \$ Mitsubishi Kagaku Institute of Life Sciences (MITILS), Machida, Tokyo 194-8511, Japan.
}

\section{Materials and Methods}

Materials. $\quad\left[2-{ }^{14} \mathrm{C}\right]$ Malonyl-CoA $(48 \mathrm{mCi} / \mathrm{mmol})$ and $\left[1-{ }^{14} \mathrm{C}\right]$ acetyl $\mathrm{CoA}(47$ $\mathrm{mCi} / \mathrm{mmol}$ ) was purchased from Moravek Biochemicals (California). Malonyl-CoA and acetyl-CoA were purchased from Sigma. 4-Coumaroyl-CoA was chemically synthesized as described previously (Abe, I.; Morita, H.; Nomura, A.; Noguchi, H. J. Am. Chem. Soc. 2000, 122, 11242-11243). Authentic samples of SEK4/SEK4b, 5,7-dihydroxy-2-methylchromone, 2,7-dihydroxy-5-methylchromone, and tetracetic acid lactone were obtained in our previous works (Abe, I.; Oguro, S.; Utsumi, Y,; Sano, Y.; Noguchi, H. J. Am. Chem. Soc. 2005, 127, 12709-12716). The CHS used in this study was cloned from young leaves of Scutellaria baicalensis. The recombinant enzyme with an additional hexahistidine tag at the C-terminal was subcloned into pET-22b(+) (Novagen), expressed in E. coli, and purified by Ni-chelate affinity chromatography as described before (Abe, I.; Morita, H.; Nomura, A.; Noguchi, H. J. Am. Chem. Soc. 2000, 122, 11242-11243). 


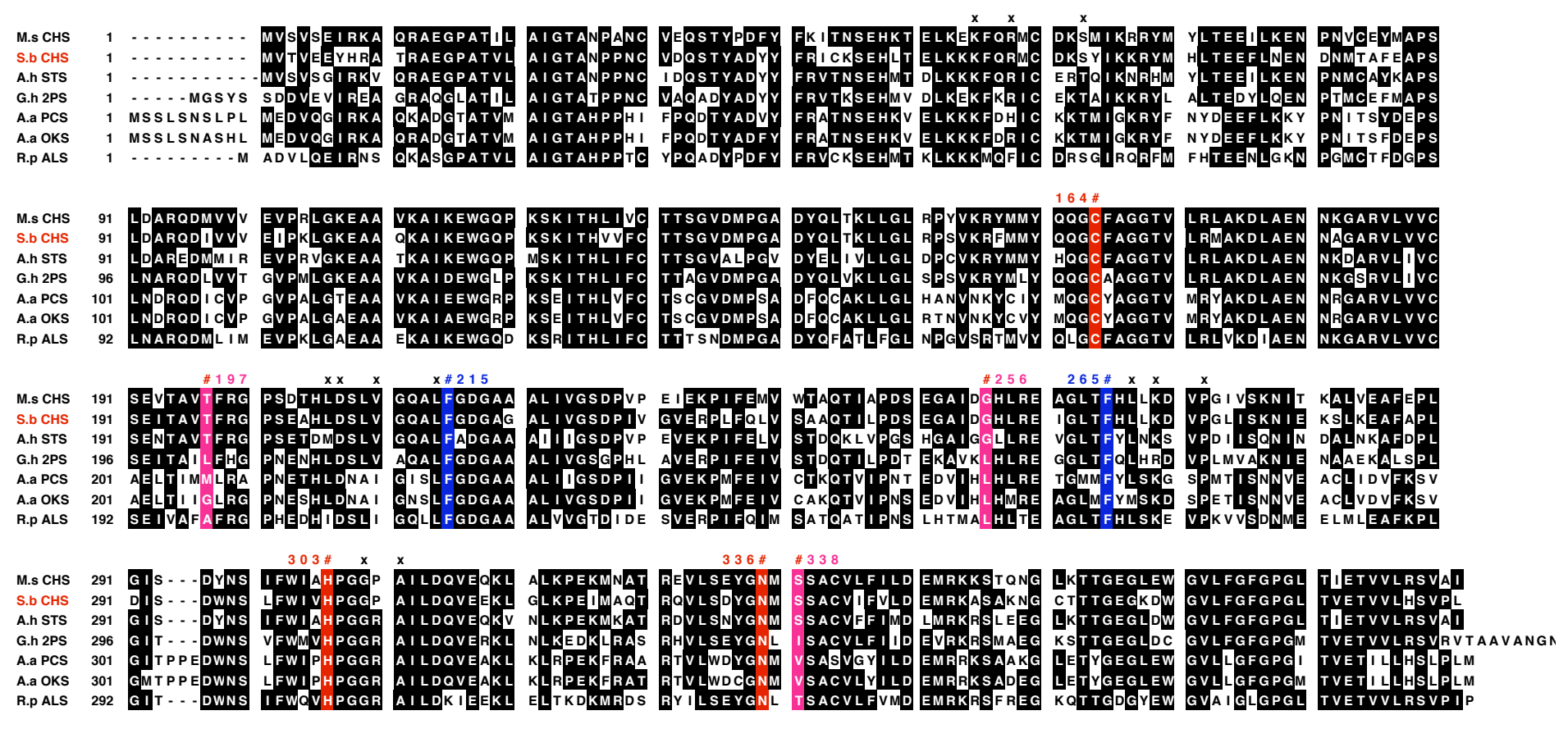

Fig. 1 Comparison of primary sequences of Scutellaria baicalensis CHS and other CHS-superfamily enzymes. M.s CHS, Medicago sativa CHS; S.b CHS, S. baicalensis CHS; A.h STS, Arachis hypogaea stilbene synthase; G.h 2PS, Gerbera hybrida 2-pyrone synthase; A.a PCS, Aloe arborescens PCS; A.a OKS, A. arborescens OKS; R.p ALS, Rheum palmatum ALS. The critical active-site residues 197, 256, and 338 (in pink), the catalytic triad (Cys164, His303, and Asn336) (in red), and the active-site Phe215 and F265 (in blue) were marked with \# (numbering in $M$. sativa $\mathrm{CHS}$ ), and residues for the CoA binding with + .

Site-Directed Mutagenesis. S. baicalensis CHS mutants (T197G, G256L, S338V, G256L/S338V，T197G/G256L，T197G/S338V，T197G/G256L/S338V，T197A/G256L/S338V, and T197M/G256L/S338V) were constructed using the QuickChange Site-Directed Mutagenesis Kit (Stratagene) and a pair of complementary mutagenic primers as follows (mutated codons are

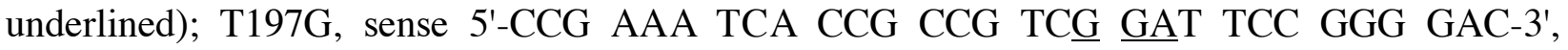

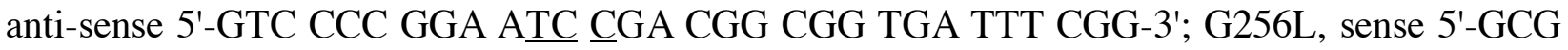

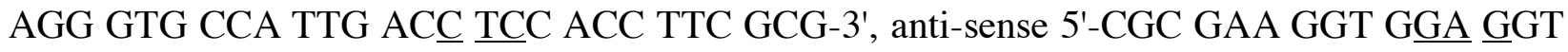

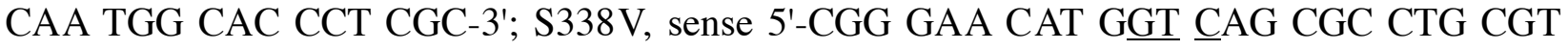
GAT CTT CG-3', anti-sense 5'-CGA AGA TCA CGC AGG CGC T므 CCA TGT TCC CG-3'; 
T197A, sense 5'-GCT CCG AAA TCA CCG CCG TCG CAT TCC GGG GAC-3', anti-sense 5'-GTC CCC GGA ATG CGA CGG CGG TGA TTT CGG AGC-3'; T197M, sense 5'-GCT CCG

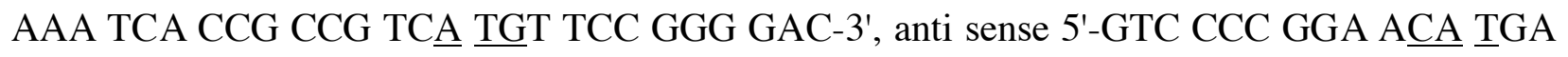
CGG CGG TGA TTT CGG AGC-3'.

Enzyme Expression and Purification. After confirmation of the sequence, the plasmid was transformed into E. coli BL21(DE3)pLysS. The cells harboring the plasmid were cultured to an $A_{600}$ of 0.6 in $\mathrm{LB}$ medium containing $100 \mu \mathrm{g} / \mathrm{mL}$ of ampicillin at $30^{\circ} \mathrm{C}$. Then, $0.4 \mathrm{mM}$ isopropyl thio- $\beta$-D-galactoside was added to induce protein expression, and the culture was incubated further at $16{ }^{\circ} \mathrm{C}$ for $14 \mathrm{~h}$. The E. coli cells were harvested by centrifugation and resuspended in $40 \mathrm{mM}$ potassium phosphate buffer, $\mathrm{pH} 7.9$, containing $0.1 \mathrm{M} \mathrm{NaCl}$. Cell lysis was carried out by sonication, and centrifuged at $15,000 \mathrm{~g}$ for $40 \mathrm{~min}$. The supernatant was passed through a column of Pro-Bond ${ }^{\mathrm{TM}}$ resin (Invitrogen) containing $\mathrm{Ni}^{2+}$ as an affinity ligand. After washing with $20 \mathrm{mM}$ potassium phosphate buffer, $\mathrm{pH} 7.9$, containing $0.5 \mathrm{M} \mathrm{NaCl}$ and 40 $\mathrm{mM}$ imidazole, the recombinant ALS was finally eluted with $15 \mathrm{mM}$ potassium phosphate buffer, pH 7.5, containing $10 \%$ glycerol and $500 \mathrm{mM}$ imidazole. Protein concentration was determined by the Bradford method (Protein Assay, Bio-Rad) with bovine serum albumin as standard.

Enzyme Reaction. The standard reaction mixture contained $27 \mathrm{nmol}$ of starter CoA (4-coumaroyl-CoA) and $54 \mathrm{nmol}$ of malonyl-CoA, and $460 \mathrm{pmol}$ of the purified recombinant enzyme in a final volume of $500 \mu \mathrm{L}$ of $100 \mathrm{mM}$ potassium phosphate buffer, $\mathrm{pH}$ 7.0. Incubations were carried out at $30{ }^{\circ} \mathrm{C}$ for 12 hours, and stopped by adding $50 \mu \mathrm{L}$ of $20 \% \mathrm{HCl}$. The products were then extracted with $1,000 \mu \mathrm{L}$ of ethyl acetate, and concentrated by $\mathrm{N}_{2}$ flow. 
The residue was dissolved in aliquot of $\mathrm{MeOH}$ containing $0.1 \%$ TFA, and separated by reverse-phase HPLC (JASCO 880) on a TSK-gel ODS-80Ts column (4.6 x $150 \mathrm{~mm}$, TOSOH) with a flow rate of $0.8 \mathrm{ml} / \mathrm{min}$. Gradient elution was performed with $\mathrm{H}_{2} \mathrm{O}$ and $\mathrm{MeOH}$, both containing 0.1\% TFA: 0-5 min, 30\% MeOH; 5-17 min, linear gradient from 30 to $60 \% \mathrm{MeOH}$; 17-25 min, $60 \% \mathrm{MeOH}$; $25-27 \mathrm{~min}$, linear gradient from 60 to $70 \% \mathrm{MeOH}$. Elutions were monitored by a multichannel UV detector (MULTI 340, JASCO) at $290 \mathrm{~nm}, 330 \mathrm{~nm}$ and $360 \mathrm{~nm}$; UV spectra (198-400 nm) were recorded every $0.4 \mathrm{~s}$. The retention time (min): SEK4 (19.3), SEK4b (20.6), 6-(2,4-dihydroxy-6-methylphenyl)-4-hydroxy-2-pyrone (16.9), 2,7-dihydroxy-5methylchromone (22.7), tetracetic acid lactone (4.4), and triacetic acid lactone (6.0).

On-line LC-ESIMS spectra were measured with a Hewlett-Packard HPLC 1100 series (Wilmington, DE) coupled to a Finnigan MAT LCQ ion trap mass spectrometer (San Jose, CA) fitted with an ESI source. HPLC separations were carried out under the same conditions as described above. The ESI capillary temperature and capillary voltage were $225^{\circ} \mathrm{C}$ and $3.0 \mathrm{~V}$, respectively. The tube lens offset was set at $20.0 \mathrm{~V}$. All spectra were obtained in both negative and positive mode; over a mass range of $\mathrm{m} / \mathrm{z} 100-500$, at a range of one scan every $2 \mathrm{~s}$. The collision gas was helium, and the relative collision energy scale was set at $30.0 \%(1.5 \mathrm{eV})$. 
(A) CHS T197G

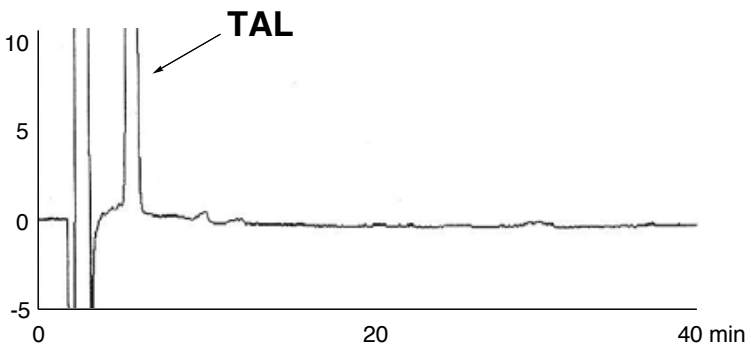

(B) CHS G256L

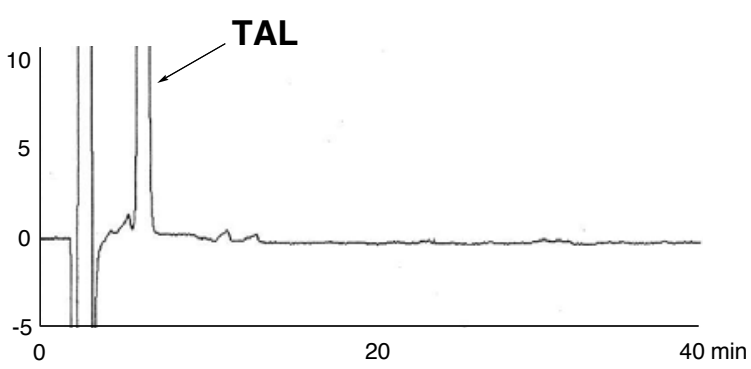

(C) CHS S338V

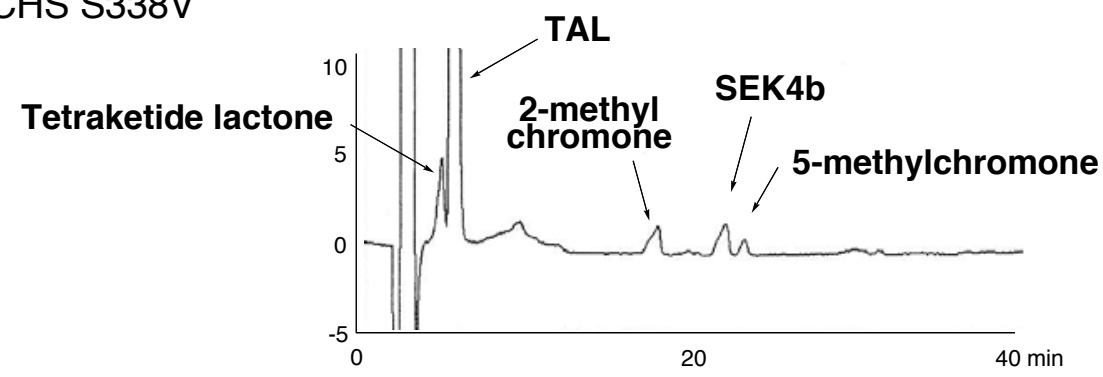

(D) CHS T197G/S338V

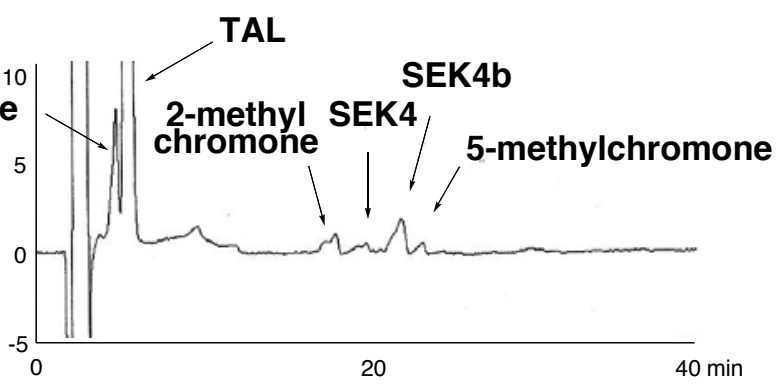

(E) CHS T197G/G256L/S338V

TAL SEK4 SEK4b

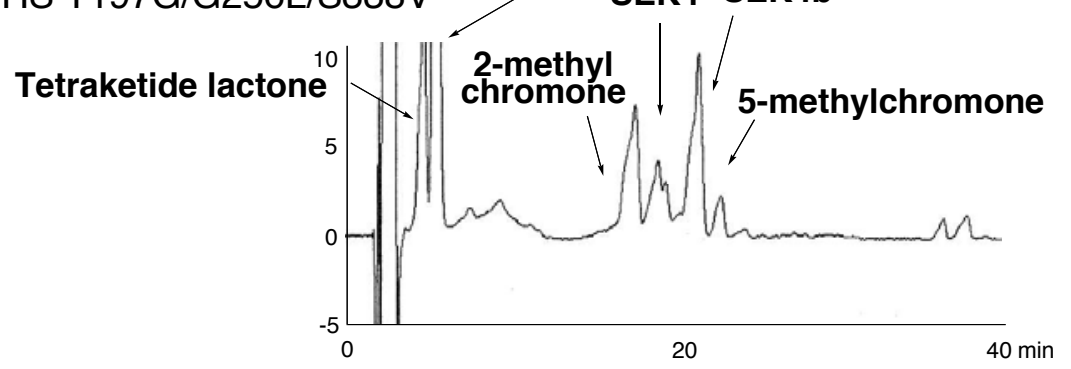

Fig. 2. HPLC elution profiles of enzyme reaction products of S. baicalensis CHS mutant; (A) T197G, (B) G256L,

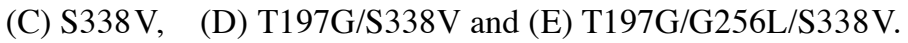


(A)
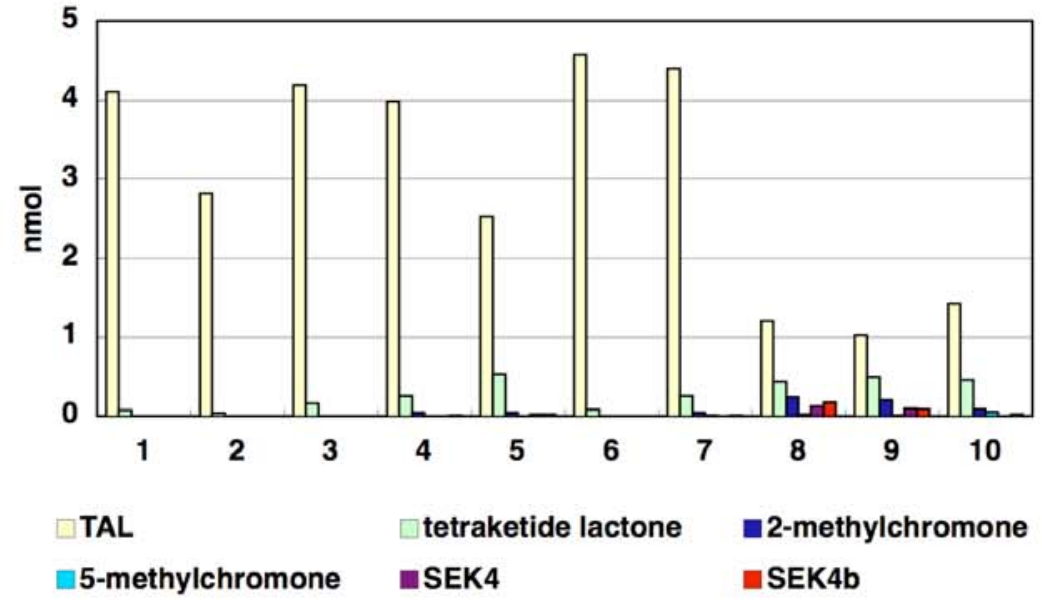

(B)

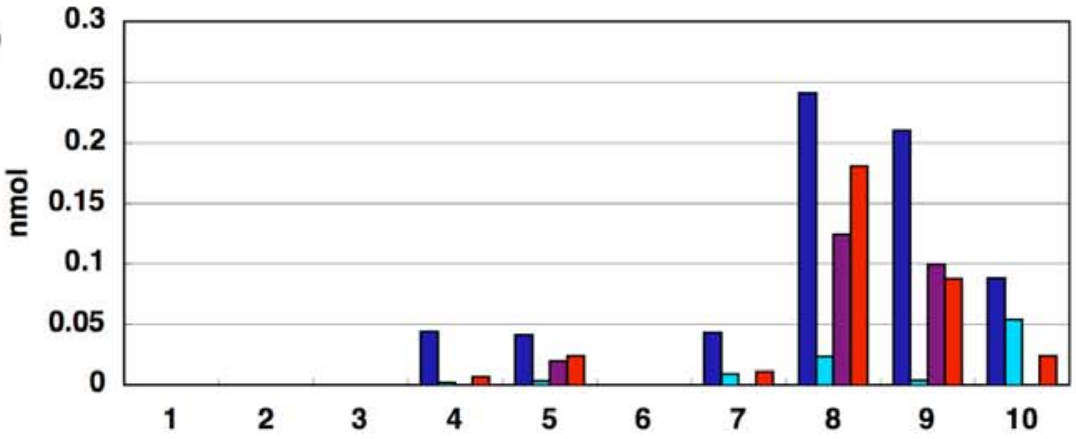

(C)

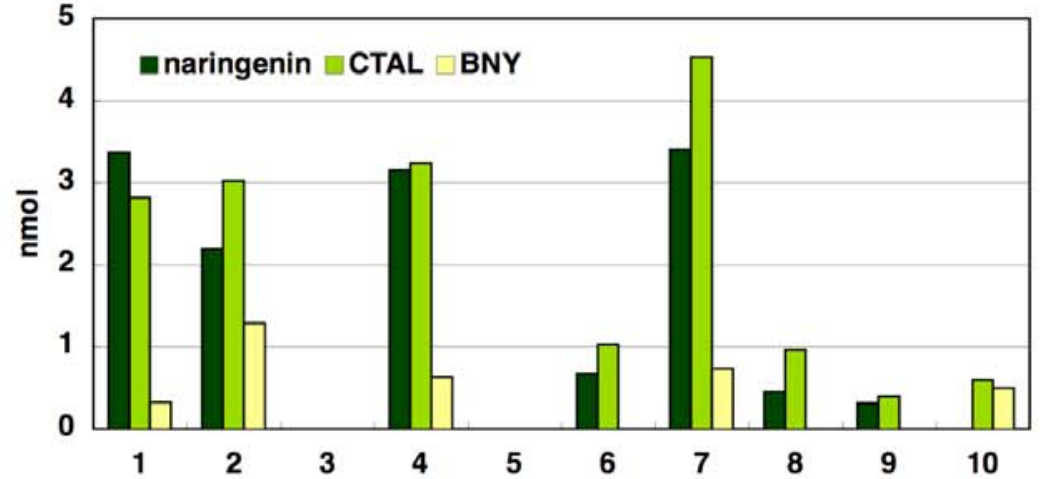

Fig. 3. Distribution pattern of polyketides produced by CHS mutants; (A) from malonyl-CoA (all products), (B) from malonyl-CoA (pentaketides and octaketides), (C) from 4-coumaroyl-CoA/malonyl-CoA. (1) wild-type, (2) T197G, (3) G256L, (4) $\underline{\mathrm{S} 338 \mathrm{~V}}$, (5) G256L/S338V, (6) T197G/G256L, (7) T197G/S338V, (8) T197G/G256L/S338V, (9) T197A/G256L/ $\underline{\mathrm{S} 338 \mathrm{~V}}$, (10) T197M/G256L/ $\underline{\mathrm{S} 338 \mathrm{~V}}$. 